\title{
Antimicrobial peptide derived from moths can eradicate UPEC biofilms and could offer a novel therapeutic option
}

$c 4$

\author{
the AMP
}

activity of

CecA has

been reported

in the past,

but efficacy

against

biofilms is a

new finding
A study published in npj Biofilms and Microbiomes has described how cecropin A (CecA), an antimicrobial peptide (AMP) derived from the greater wax moth Galleria mellonella, can destroy biofilm-forming uropathogenic Escherichia coli (UPEC) cells and acts synergistically with the antibiotic nalidixic acid (NAL).

Urinary tract infections present considerable clinical challenges - not only because of their prevalence but also because resistance develops to commonly used antibiotics. UPEC are particularly hard to eradicate because they can form biofilms on epithelial cells and urinary catheters and have developed a number of resistance mechanisms against antibiotics, such as fimbriae and an impenetrable extracellular matrix (ECM). However, the mechanisms of action of AMPs are different to classical antibiotics, which means that AMPs might be able to bypass the resistance mechanisms of UPEC. Furthermore, this ability also raises the possibility that AMPs could act synergistically with antibiotics for a double-pronged attack on infections.

In order to investigate this phenomenon, Kalsy and colleagues screened insect-derived AMPs for activity against UPEC. During this screen, they discovered that CecA had activity against UPEC biofilms. Interestingly, the AMP activity of CecA has been reported in the past, but efficacy against biofilms is a new finding. "Although cecropins were the first insect AMPs to be discovered and are known to kill Gram-negative bacteria by forming membrane pores, this specific biofilm-busting activity had not been reported or investigated before," corresponding author Krishnendu Mukherjee tells Nature Reviews Urology. Thus, the team went on to further investigate this finding using combinations of CecA with NAL in a checkerboard assay to uncover synergistic effects. Using this approach, absolute minimum inhibitory concentration (MIC) for CecA decreased from $100 \mu \mathrm{g} / \mathrm{ml}$ to $50 \mu \mathrm{g} / \mathrm{ml}$, and for NAL decreased from $60 \mu \mathrm{g} / \mathrm{ml}$ to $0.5 \mathrm{ng} / \mathrm{ml}$, indicating synergy. No toxicity was seen in erythrocytes or fibroblasts at $50 \mu \mathrm{g} / \mathrm{ml} \mathrm{CecA}$, but at $75 \mu \mathrm{g} / \mathrm{ml}$ some cytotoxicity was observed.

A combination dose of $50 \mu \mathrm{g} / \mathrm{ml}$ $\mathrm{CecA}+0.5 \mathrm{ng} / \mathrm{ml}$ NAL was, therefore, chosen for further analysis as it is the minimum dose of each agent that is ineffective as monotherapy but that demonstrates synergy to completely inhibit the growth of planktonic UPEC cells. In an in vivo model using G. mellonella larvae infected with the UPEC strain CFT073, $\mathrm{CecA} \pm$ NAL slightly improved survival of CFT073-infected larvae, even though NAL treatment alone was ineffective. Overall, CFT073 survival in infected larvae was reduced more significantly by the combination treatment than either agent alone.

Mechanisms were investigated using SYTO9, which stains intact membranes of living cells green, and propidium iodide, which specifically penetrates cells with
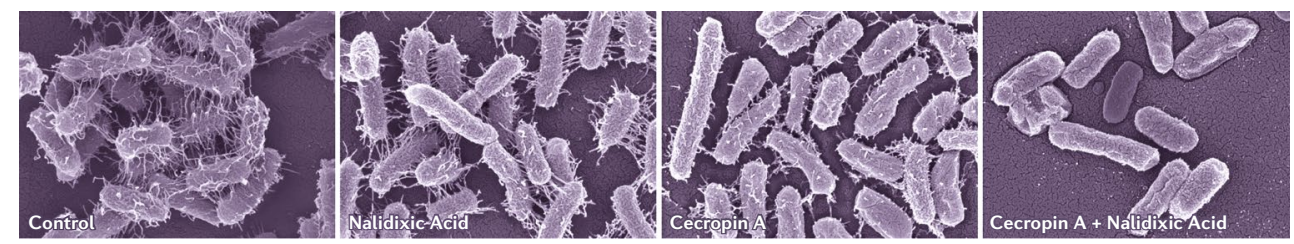

Credit: Image courtesy of M. Hardt, Justus-Liebig-Universität Gießen, Germany.

damaged membranes and stains them red. The proportion of red cells increased progressively with NAL, CecA and CecA + NAL treatment, and loss of membrane integrity was observed after CecA administration. At the final concentration range of $0.25 \mu \mathrm{M}-1 \mu \mathrm{M}$ CecA, up to $15 \%$ permeabilization of the bacterial membrane was observed. Electron microscopy techniques revealed that CecA + NAL prevented CFT073 biofilm formation by targeting type I and $P$ fimbriae, which enable attachment of biofilm-forming cells to surfaces and reduce contact between AMPs and bacterial membranes. Other effects of these agents included changes in the structure of the ECM, cell envelope and cytoplasmic density of the UPEC cells compared with control treatments. Damage was greatest for the combination treatment, once again indicating synergy, possibly owing to CecA improving NAL entry into bacterial cells.

In a clinical landscape in which antibiotic resistance continues to be a problem, nonantibiotic approaches to infection are urgently needed, an area that the team plans to continue working in: "We plan to investigate new biofilm-degrading, insectderived antimicrobial compounds that are non-toxic to humans," comments Mukherjee, "and we are also interested in developing a robust drug-delivery system for treating biofilms infection by UPEC and other biofilm-forming resistant bacteria in humans, using insect AMPs such as CecA."

Annette Fenner

ORIGINAL ARTICLE Kalsy, M. et al. The insect antimicrobial peptide cecropin A disrupts uropathogenic Escherichia coli biofilms. npj Biofilms Microbiomes 6, 6 (2020) RELATED ARTICLE Sihra, N. et al. Nonantibiotic prevention and management of recurrent urinary tract infection. Nat. Rev. Urol. 15, 750-776 (2018) 